\title{
La formation des enseignants sur la diversité et les rapports ethniques : regard comparatif France, Québec, Belgique et Suisse
}

\section{Teacher training on diversity and ethnic relations: comparative perspective of France, Quebec, Belgium and Switzerland \\ Teacher training on diversity and ethnic relations: comparative perspective of France, Quebec, Belgium and Switzerland}

\author{
Maryse Potvin, Fabrice Dhume, Marie Verhoeven et Tania Ogay
}

Volume 46, numéro 2, automne 2018

Pluralisme, équité et rapports ethniques dans la formation du personnel des milieux éducatifs

URI : https://id.erudit.org/iderudit/1055560ar

DOI : https://doi.org/10.7202/1055560ar

Aller au sommaire du numéro

Éditeur(s)

Association canadienne d'éducation de langue française

ISSN

1916-8659 (numérique)

Découvrir la revue

Citer cet article

Potvin, M., Dhume, F., Verhoeven, M. \& Ogay, T. (2018). La formation des enseignants sur la diversité et les rapports ethniques : regard comparatif France, Québec, Belgique et Suisse. Éducation et francophonie, 46(2), 30-50. https://doi.org/10.7202/1055560ar

\section{Résumé de l'article}

La formation et l'évaluation des compétences des enseignants pour agir en contexte de diversité interpellent autant les décideurs gouvernementaux que les milieux universitaires et scolaires. Cet article porte un regard comparatif transversal sur la formation du personnel scolaire en matière de diversité, d'équité et des rapports ethniques en éducation en France, au Québec, en Belgique francophone et en Suisse, notamment dans les politiques, référentiels de compétences, dispositifs, programmes, cours et contenus de formation. Ce regard comparatif sur quatre sociétés de la francophonie montre que chaque contexte a développé un discours, des orientations et des dispositifs de formation assez différents sur ces questions, mais quand même réunis par certaines lignes de convergence.

Tous droits réservés @ Association canadienne d'éducation de langue française, Ce document est protégé par la loi sur le droit d’auteur. L’utilisation des 2018 services d'Érudit (y compris la reproduction) est assujettie à sa politique d'utilisation que vous pouvez consulter en ligne.

https://apropos.erudit.org/fr/usagers/politique-dutilisation/ 


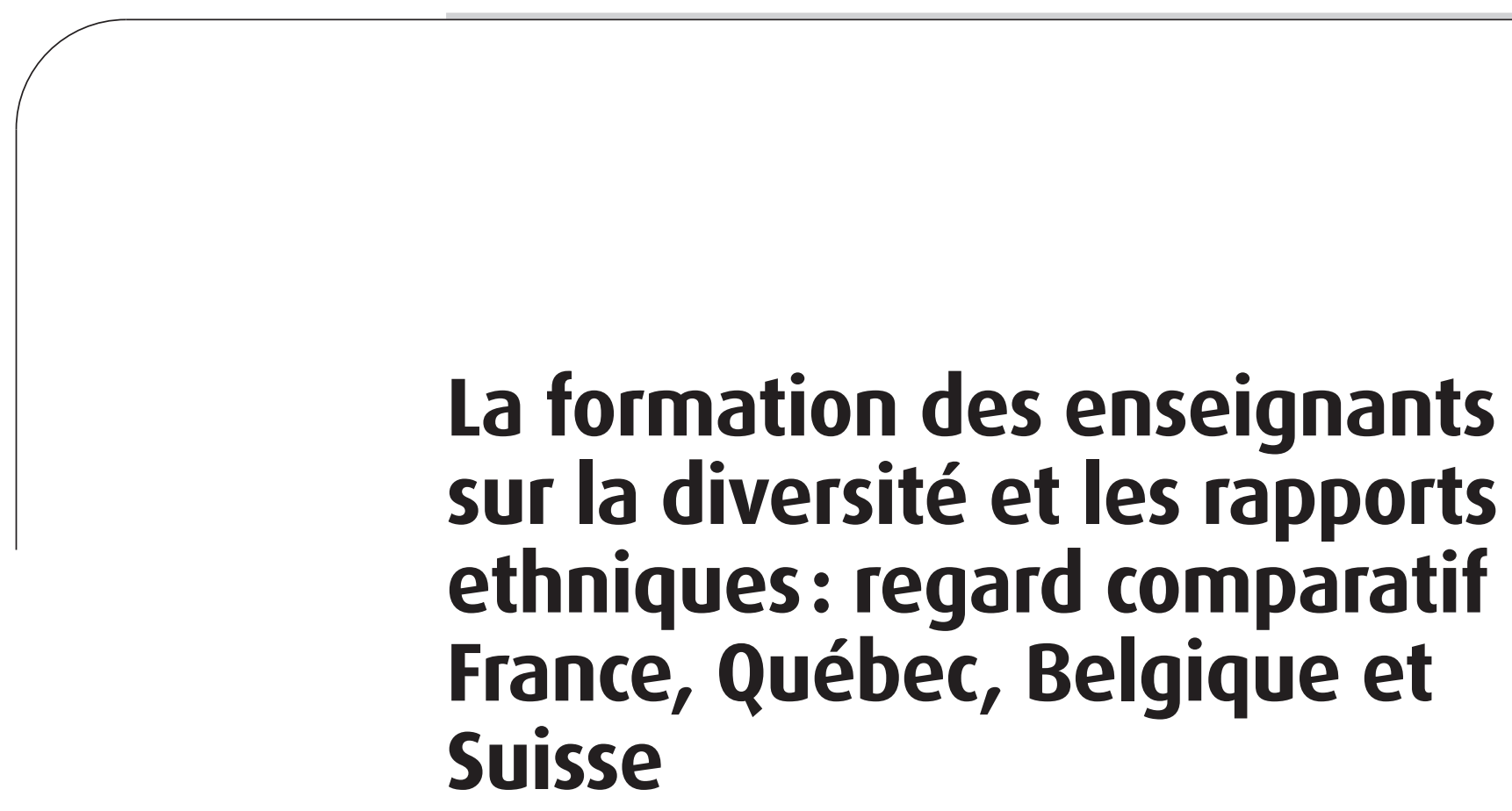

Citer cet article :

POTVIN, M., DHUME, F., VERHOEVEN, M. et OGAY, Tania (2018). La formation des enseignants sur la diversité et les rapports ethniques: regard comparatif France, Québec, Belgique et Suisse. Éducation et francophonie, 46(2), 30-50.
Maryse POTVIN

Université du Québec à Montréal, Québec, Canada

\section{Fabrice DHUME}

Université de Paris Diderot / URMIS, Paris, France

Marie VERHOEVEN

Université Catholique de Louvain / GIRSEF-IACCHOS, Louvain-la-neuve, Belgique

\section{Tania OGAY}

Université de Fribourg, Fribourg, Suisse

\section{RÉSUMÉ}

La formation et l'évaluation des compétences des enseignants pour agir en contexte de diversité interpellent autant les décideurs gouvernementaux que les milieux universitaires et scolaires. Cet article porte un regard comparatif transversal sur la formation du personnel scolaire en matière de diversité, d'équité et des rapports ethniques en éducation en France, au Québec, en Belgique francophone et en Suisse, notamment dans les politiques, référentiels de compétences, dispositifs, programmes, 
cours et contenus de formation. Ce regard comparatif sur quatre sociétés de la francophonie montre que chaque contexte a développé un discours, des orientations et des dispositifs de formation assez différents sur ces questions, mais quand même réunis par certaines lignes de convergence.

\section{ABSTRACT}

Teacher training on diversity and ethnic relations: comparative perspective of France, Quebec, Belgium and Switzerland

Maryse POTVIN, University of Quebec in Montreal, Quebec, Canada

Fabrice DHUME, University of Paris Diderot / URMIS, Paris, France

Marie VERHOEVEN, Catholic University of Louvain / GIRSEF-IACCHOS, Louvain-la-neuve, Belgium

Tania OGAY, University of Friborg, Friborg, Switzerland

The training and assessment of teachers' competencies for working in a context of diversity concerns both government decision-makers and educational institutions. This article provides a cross-sectional comparative view on the training of school personnel on diversity, equity and ethnic relations in France, Quebec, French-speaking Belgium and Switzerland, in particular on policies, skill frameworks, programs, courses and training content. This comparative view of four French-speaking societies shows that each context has developed a discourse on these issues with very different orientations and training methods, but are still united by certain lines of convergence.

\section{RESUMEN}

\section{Teacher training on diversity and ethnic relations: comparative perspec- tive of France, Quebec, Belgium and Switzerland}

Maryse POTVIN, University of Quebec in Montreal, Quebec, Canada

Fabrice DHUME, University of Paris Diderot / URMIS, Paris, France

Marie VERHOEVEN, Catholic University of Louvain / GIRSEF-IACCHOS, Louvain-la-neuve, Belgium

Tania OGAY, University of Friborg, Friborg, Switzerland

The training and assessment of teachers' competencies for working in a context of diversity concerns both government decision-makers and educational institutions. This article provides a cross-sectional comparative view on the training of school personnel on diversity, equity and ethnic relations in France, Quebec, French-speaking Belgium and Switzerland, in particular on policies, skill frameworks, programs, 
courses and training content. This comparative view of four French-speaking societies shows that each context has developed a discourse on these issues with very different orientations and training methods, but are still united by certain lines of convergence.

\section{INTRODUCTION}

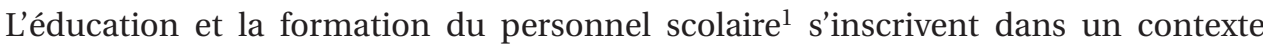
global marqué par la mondialisation et la diversification des populations scolaires (ONU, 2010). L'adaptation des systèmes scolaires à la diversité ethnoculturelle ${ }^{2}$ repose sur la préparation des acteurs à adopter des pratiques d'équité et à former de futurs citoyens à vivre ensemble, et divers phénomènes ont partout accru un sentiment d'urgence à cet égard: débats tendus sur la religion, arrivée de réfugiés, attentats, montée du racisme et de la radicalisation. L'acquisition de compétences (cognitives, affectives, sociales, éthiques, professionnelles) par le personnel scolaire pour relever ces défis fait l'objet d'un large consensus international et scientifique (Arnesen et al., 2009; UNESCO, 2009, 2015). Depuis les rapports Faure (1978) et Delors (1996), l'ONU a développé plusieurs plans d'action, cadres de référence et programmes incitant les États à développer des politiques et des curriculums pour la formation des enseignants, fondés sur diverses approches (et paradigmes), dont l'éducation interculturelle (la différence culturelle), inclusive (l'équité), à la citoyenneté démocratique, globale et aux droits (les valeurs civiques communes), critique, antiraciste ou antidiscriminatoire (l'égalité substantive, la justice sociale) (Potvin et Larochelle-Audet, 2016). Cette diversité d'approches montre qu'en dépit de préoccupations communes, le débat reste ouvert quant aux réponses à y apporter et à leur application réelle dans chaque contexte.

Cet article dresse un portrait comparatif de la formation initiale du personnel scolaire sur la diversité, l'équité et les rapports ethniques en France, au Québec, en Belgique francophone et en Suisse ${ }^{3}$. Ces quatre sociétés ont des histoires et des conceptions bien différentes de la «nation», de la Politique de reconnaissance de la diversité

1. Le terme "personnel scolaire» inclut ici les enseignants et les autres corps professionnels des milieux scolaires.

2. Liée dans une large part aux mouvements migratoires, à la colonisation ou aux conquêtes, les notions de diversité "ethnoculturelle», "religieuse» et "linguistique»- parfois désignées ici par les termes "diversité», "diversité culturelle» ou «diversité ethnoculturelle» - font référence au pluralisme de fait, aux rapports inégaux de pouvoir entre majoritaires et minoritaires et aux expériences spécifiques qui en découlent. Cette diversité réunit ici des marqueurs comme la langue, la religion, la "race», la couleur, l'origine ethnique ou nationale, le statut d'immigrant ou de réfugié. Ces marqueurs sont appréhendés selon une approche constructiviste et intersectionnelle, comme des expériences reposant sur des rapports d'oppression fondés sur divers marqueurs de la diversité sociale (genre, condition sociale, etc.) (Potvin et al, 2015).

3. Cet article se penche sur la partie francophone dans chaque pays. 
(Taylor, 1992), des rapports entre majoritaires et minoritaires et du rôle de l'éducation sur ces enjeux. Pour des raisons historiques distinctes, elles ont connu une adaptation récente de leurs institutions publiques à la diversité, en dépit d'un pluralisme de longue date. Adoptant une démarche comparative, l'article situe d'abord la place de la diversité dans les politiques éducatives de ces sociétés, puis dans leurs dispositifs de formation du personnel scolaire, notamment les référentiels de compétences, les programmes et les cours. Il s'interroge ensuite sur les effets de cette formation sur les pratiques enseignantes et revient en conclusion sur les convergences entre les contextes, afin de soulever des pistes pour la formation et la recherche.

\section{La place de la diversité dans les politiques éducatives}

Les politiques des quatre sociétés sont très différentes en matière de formation du personnel à la diversité. En France, la «diversité ethnoculturelle» n'est pas une catégorie de l'action scolaire, eu égard à la référence unitariste de l'imaginaire républicain (Zoïa, 2015). À l'école, la notion de «diversité» renvoie aux «problèmes» implicitement associés aux différences ethnoreligieuses des publics (vus comme des immigrés), à «l'hétérogénéité» des milieux (violence, tensions), ou à l'adaptation aux «handicaps» (Lantheaume, 2011; Dhume, 2012). Sous cette conception colorblind et assimilationniste des enjeux scolaires, des exceptions existent envers des territoires (outre-mer...) ou des populations institutionnellement altérisées («gens du voyage»...), mais dont le modèle recycle en partie l’héritage colonial (Falaize, 2016).

La situation actuelle montre une absence de problématisation politique cohérente et assumée. L'interculturalité a été, dans les années 1970, le discours de certains réseaux enseignants engagés dans les dispositifs pour les "enfants de migrants» 4 , et s'appuyant sur les théories psychoculturelles, parfois essentialistes (Sayad, 2014; Dhume et al., 2011). Des enseignements de langues et cultures d'origine, organisés de façon périphérique à l'école, ont survécu jusqu'à aujourd'hui, bien que constamment stigmatisés (Lorcerie, 1994) par une pression normative croissante visant la «maîtrise du français» et «l'adhésion aux valeurs de la République». En 1981, la création des Zones d'éducation prioritaire (ZEP) ramène au premier plan l'enjeu d'une égalité réelle, mais cette nouvelle focalisation territorialisée maintient la dominance d'un discours sur les inégalités sociales, occultant les rapports ethniques tout en ethnicisant les problèmes (Morel, 2002). Instrumentalisée par la polémique sur le voile depuis 1989, l'école est requalifiée de "sanctuaire républicain», afin de justifier la prohibition des signes religieux «ostentatoires». Au profit d'un discours de "défense des valeurs de la République», la laïcité est aujourd'hui «la plus efficace métonymie de l'islamophobie» (Lorcerie, 2012). Depuis la fin des années 1980, la diversité est

4. Comme la revue Migrants-formation ou les classes d'initiations. Créés en 1975, les Centres de formation et d'information pour la scolarisation des enfants de migrants (CEFISEM) ont vus en 1990 s'élargir leurs missions à l'accompagnement des Zones d'éducation prioritaire, avant de devenir en 2002 le Centre académique pour la scolarisation des enfants allophones nouvellement arrivés et des enfants issus de familles itinérantes et de voyageurs (CASNAV). 
l'objet d'une gestion négative, l'interdiction des signes religieux des élèves en 2004 étant la principale mesure adoptée sur ces enjeux.

Le ministère de l'Éducation nationale a souvent opté pour un discours neutralisé, dépolitisant les thèmes jugés sensibles, comme la lutte contre les discriminations, pourtant érigée en priorité officielle en 2008. L'usage progressif du terme «diversité» dans le discours de l'institution depuis la Loi d'orientation sur l'éducation de 1989, et surtout après 2004 (la "promotion de la diversité» se substitue à la «lutte contre les discriminations»), relève d'une préoccupation consensuelle plus que d'une reconnaissance politique. En réponse aux attentats de janvier 2015, notamment contre Charlie hebdo, une timide inflexion se manifeste envers l'égalité et les discriminations, mais le discours ne rompt pas avec l'ambiguïté qui caractérise la reconnaissance de ces enjeux dans le cadre scolaire (Dhume, 2016).

Au Québec, province francophone au sein d'une fédération plurinationale, multiculturelle et bilingue (Kymlicka, 1998), ces enjeux sont bien inscrits dans les politiques publiques, notamment en éducation, une compétence exclusive aux provinces. L'immigration étant de longue date un objet de "concurrence de loyautés» dans le conflit historique qui oppose les peuples dits «fondateurs» (Mc Roberts, 1999; Potvin, 2016), l'intégration des immigrants à la majorité francophone «fragile» (car historiquement minorisée) est une réalité relativement nouvelle sur le plan de sa gestion étatique et de ses politiques publiques (Mc Andrew, 2010). Jusqu'aux années 1960, les immigrants fréquentent l'école anglaise à $90 \%$ et s'intègrent au groupe dominant anglophone, qui contrôle alors l'économie (Levine, 1990). L'adoption de la Charte de la langue française (1977), qui fait du français la langue officielle du Québec et amène les élèves immigrants arrivés après 1977, et leurs descendants, à fréquenter obligatoirement l'école française ${ }^{5}$, marque le passage des francophones au statut sociologique de «groupe majoritaire» au Québec.

À la même époque, le gouvernement du Québec élabore la Politique québécoise du développement culturel (1978), qui pose un modèle interculturel d'intégration autour d'une «culture de convergence française». En milieu solaire, cette dominance du paradigme interculturel repose sur une «fusion de perspectives» (Ouellet, 1991), mais le ministère de l'Éducation (Ministère de l'Éducation du Québec, 1985) préconise surtout l'adoption de mesures compensatoires, vues comme de l'éducation interculturelle. Dans les syndicats d'enseignants ${ }^{6}$ et le réseau scolaire anglophone, la posture est beaucoup plus critique sur l'école. On reproche à l'interculturel de voir la diversité comme des différences "à gérer», de les considérer de manière figée et binaire, et d'enseigner aux élèves à les accepter sans questionner leur construction sociale,

5. Par contre, il existe un réseau d'écoles anglaises et un réseau d'écoles privées, aussi fréquentés par les élèves issus de l'immigration (surtout à la $2^{\mathrm{e}}$ génération) (McAndrew et Bakhshaei, 2016), dont 61 écoles dites «ethnospécifiques» (à majorité juives) (Tremblay, 2016).

6. Dont la Corporation des enseignants du Québec (CEQ) et ses Cahiers de pédagogie progressiste. 
rendant invisible l'articulation des systèmes de domination (racisme, sexisme, classisme) dans la construction identitaire des élèves (Potvin, 2018).

Au cours des années 1990, les perspectives critiques disparaissent et la citoyenneté s'impose comme un concept-clé dans les discours normatifs pour gérer «la fragmentation sociale» (Labelle et Salée, 1999). Au Québec, le discours public vise alors l'intégration "à la majorité francophone» et, avec l'Énoncé de politique en matière d'immigration et d'intégration (Ministère des Communautés culturelles et de l'Immigration, 1990), les immigrants ont le «contrat moral» de respecter les valeurs-piliers de la société (langue française, démocratie, droits et libertés). En milieu éducatif, les questions interculturelles sont dirigées vers les conditions de la «cohésion sociale». Au début des années 2000, le paradigme civique s'institutionnalise par l'introduction de l'éducation à la citoyenneté dans le curriculum, et la Politique d'intégration scolaire et d'éducation interculturelle (Ministère de l'Éducation du Québec, 1998), toujours en vigueur, articule interculturalisme et citoyenneté. Axée sur l'intégration des immigrants, le partage d'une langue publique commune et le développement d'une citoyenneté pluraliste et démocratique, l'une des orientations de cette politique cible spécifiquement la formation «théorique et pratique [...] de tout le personnel des établissements d'enseignement [...] pour relever les défis éducatifs liés, d'une part, à la diversité ethnoculturelle, linguistique et religieuse des effectifs et, d'autre part, à la nécessaire socialisation commune de l'ensemble des élèves» (Ministère de l'Éducation du Québec, 1998, p. 32-33). Enfin, la nouvelle Politique de la réussite éducative (2017) repose sur le paradigme de l'équité et articule des objectifs de lutte contre les discriminations et les inégalités, de prise en compte des réalités socioculturelles de l'élève, et de développement de connaissances et de compétences civiques, démocratiques et de justice.

Le rapport à la diversité ethnoculturelle en Belgique est aussi caractérisé par une «double complexité», puisqu'il s'agit d'un État plurinational, marqué par le conflit central entre la Flandre (néerlandophone) et la Wallonie (francophone), et polyethnique, en lien avec les vagues migratoires successives. Leurs politiques éducatives respectives concernant les élèves issus de l'immigration diffèrent et révèlent des orientations contrastées. En tant que «majorité fragile», la Flandre a adopté des politiques et programmes relevant à la fois d'un «multiculturalisme volontariste» (Adam, 2011) (ex.: les politiques de discrimination positive) et d'un assimilationnisme linguistique et culturel (ex.: les programmes de "parcours d'intégration»). En revanche, la domination culturelle historique de la Wallonie a contribué à maintenir une forme d'indifférence envers les enjeux ethniques, renforcée par l'influence du modèle français républicain. Comme en France, la diversité ethnoculturelle a longtemps été occultée pour ensuite émerger dans l'espace public comme un "problème» ou un 
«handicap socioculturel» (Martiniello et Manço, 1993) ${ }^{7}$. Les politiques éducatives ont été tardivement sensibles à ces questions. Dans les années 1980 et 1990, des programmes promouvant la diversité linguistique et la pédagogie interculturelle ont vu le jour (Adam, 2011), en restant confinés à des initiatives locales dans «les écoles à forte concentration" (Martiniello et Manço, 1993). Aujourd'hui, les politiques éducatives en Fédération Wallonie-Bruxelles continuent d'aborder la diversité ethnoculturelle sous l'angle des inégalités sociales. Les politiques compensatoires - des Zones d'éducation prioritaires mises en place en 1989 jusqu'aux derniers décrets proposant un financement différencié aux établissements défavorisés - sont basées sur une approche déficitaire qui prend en compte les indicateurs socioéconomiques, mais qui rendent invisibles les enjeux ethnoculturels (Martiniello et Manço, 1993; Verhoeven, 2003). Les projets d'éducation interculturelle ou d'ouverture aux langues et cultures d'origine continuent de s'organiser sur la base d'appels à projets auxquels répondent les établissements, sans donner lieu à une politique inclusive plus ambitieuse.

La formation des enseignants reflète cette réticence à considérer ces enjeux comme une question centrale, et non comme un "problème lié à certains contextes". L'influence des questions sociales et ethniques sur l'école et la pratique enseignante occupe une place très marginale dans la formation des futurs enseignants, centrée sur les aspects disciplinaires ou pédagogiques. Si l'égalité des chances de réussite et la lutte contre les discriminations sont présentes (et renforcées depuis les années 1990 par les décrets), la traduction de ces objectifs dans la formation des futurs enseignants est en deçà de ces ambitions.

En Suisse, l'attention portée à la diversité « historique», liée à la coexistence de quatre groupes nationaux occupant des régions linguistiques subdivisées en cantons dans une fédération plurinationale, engendre une forme de marginalisation des questions liées à la diversité découlant des migrations internationales plus récentes, diversité pensée avant tout comme "sociale» et «culturo-religieuse», et pour laquelle peu de moyens sont consacrés (Ogay, 2012). Dans un texte des ministres cantonaux francophones et italophones, on retrouve les mêmes objectifs qu'en Belgique quant aux «finalités et objectifs de l'école publique» (Conférence intercantonale de l'instruction publique de la Suisse romande et du Tessin, 2003), mais leur traduction dans les curriculums de formation des enseignants est encore très modeste, se heurtant notamment à la souveraineté des cantons en matière d'éducation et de formation des enseignants. Une proposition de curriculum commun pour la formation des enseignants à la diversité culturelle a bien été formulée par un groupe d'experts (Lanfranchi, Perregaux et Thommen, 2000), mandatés par la Conférence des ministres cantonaux de l'éducation, mais elle n'a donné lieu qu'à une simple recommandation (non

7. Par contre, le secteur " officiel» des écoles publiques est plus marqué par un modèle d'intégration civique et universaliste, alors que le secteur catholique (écoles libres et confessionnelles subventionnées), se caractérisent par un "personnalisme chrétien » qui aborde les différences selon une conception dépolitisée de la citoyenneté multiculturelle (Verhoeven, 2003). 
contraignante) de la Conférence des recteurs et rectrices des hautes écoles pédagogiques (2007). Un groupe de travail émanant de cette conférence a ensuite défini un cadre commun de formation interculturelle des enseignants, mais ses efforts n'ont pas abouti (Ogay, 2018). Si la prise en compte de la diversité culturelle dans la formation des enseignants mobilise de nombreux chercheurs et formateurs depuis les années 1970 (Rey-von Allmen, 2011), les mesures institutionnelles demeurent inexistantes.

\section{Des référentiels de compétences peu arrimés aux programmes de formation}

En France, la formation des enseignants est organisée depuis 2013 par les Écoles supérieures du professorat et de l'éducation (ESPE), qui délivrent une formation sanctionnée par le diplôme de Master «Métiers de l'enseignement, de l'éducation et de la formation» (MEEF), pour les différents ordres d'enseignement. La formation, qui dure deux ans, est organisée autour du concours d'accès à la fonction publique en fin de première année, et occupée à $50 \%$ par un stage en seconde année. Le ministère accrédite les ESPE en fonction d'un Cadre national des formations dispensées au sein des masters MEEF (2013), qui mentionne à l'article 2 l'acquisition des compétences nécessaires à la pratique, de même qu'un tronc commun de formation pour tous les étudiants, portant autant sur les gestes professionnels liés aux situations d'apprentissage (dont la différenciation et la prise en compte de la diversité des publics), que sur les principes éthiques du métier (enseignement de la laïcité, lutte contre les discriminations, égalité des sexes). Ces thèmes peuvent être abordés selon une approche disciplinaire et pluridisciplinaire.

Depuis le début des années 2000, et l'introduction de l'approche par compétences, les questions d'immigration, de langue et de discriminations sont donc à la fois intégrées dans les référentiels généraux de formation des élèves et dans le Référentiel de compétences des métiers du professorat et de l'éducation (Ministère de l'Éducation nationale, 2013) qui indique des attendus professionnels, sans clarifier les implicites de la notion de diversité. Ces questions apparaissent dans quatre des quatorze compétences, communes à tous les personnels d'éducation, qui relèvent du tronc commun de formation. Outre deux compétences pédagogiques fondamentales, la $\mathrm{n}^{\circ} 3$ "Connaître les élèves et les processus d'apprentissage » et la nº 4 «Prendre en compte la diversité des élèves", d'autres compétences d'ordre éthique comportent des composantes très explicites, dont la $n^{\circ} 6$ "Agir en éducateur responsable et selon des principes éthiques», qui nécessite d' "éviter toute forme de dévalorisation à l'égard des élèves, des parents, des pairs ", de "se mobiliser et mobiliser les élèves contre les stéréotypes et les discriminations de tout ordre», et de "prévenir et gérer les violences scolaires, identifier toute forme d'exclusion ou de discrimination [...]». De même, la no 8 «Utiliser une langue vivante étrangère dans les situations exigées par son métier», invite à «participer au développement d'une compétence interculturelle chez les élèves». Il est toutefois attendu des enseignants un travail d'interprétation et 
de synthèse, du fait de la faible institutionnalisation des compétences dans les programmes de formation.

Au Québec, la formation initiale est assurée par les universités dans des programmes de quatre ans menant au brevet d'enseignement. Depuis la réforme du début des années 2000, la structure de la formation initiale s'appuie sur l'approche par compétences. Le ministère de l'Éducation fixe les grandes orientations que doivent respecter les programmes universitaires afin d'être accrédités par le Comité d'agrément des programmes de formation à l'enseignement, qui évalue périodiquement ces programmes. Le référentiel ministériel de compétences professionnelles des enseignants (Ministère de l'Éducation du Québec, 2001) ${ }^{8}$ sert de cadre aux universités dans l'élaboration de leurs programmes et dans l'évaluation des acquis des étudiants au cours de leur formation théorique et pratique (un stage par année). Il définit le concept de "compétence» comme un ensemble de ressources mobilisées en action (savoirs, savoir-faire et savoir-être) et constituant un «savoir-agir en contexte». Il repose sur douze compétences, regroupées en quatre catégories (fondement, acte d'enseigner, contexte social et scolaire, identité professionnelle), mais aucune ne porte explicitement sur la diversité ethnoculturelle (Potvin et al., 2015). Les finalités d'une éducation interculturelle sont très implicites dans certaines compétences, dont la $n^{\circ} 1$ «Agir en tant que professionnel héritier, critique et interprète d'objets de savoirs ou de culture dans l'exercice de ses fonctions", la no 3 «Concevoir des situations d'enseignement-apprentissage pour les contenus à faire apprendre en fonction des élèves concernés [...] », la nº 6 «Planifier, organiser et superviser le mode de fonctionnement du groupe-classe en vue de favoriser l'apprentissage et la socialisation des élèves », et la no 12 «Agir de façon éthique et responsable [...]».

En Belgique, la formation des futurs enseignants est partagée entre les Hautes Écoles, chargées de l'enseignement supérieur pédagogique des enseignants de la maternelle, du primaire et du cycle du secondaire inférieur (formation de trois ans), et les universités, chargées de former les enseignants du secondaire supérieur. Ces derniers acquièrent une formation en didactique de trente crédits - l'équivalent d'une demi-année d'études - complémentaire à leur master disciplinaire, soit en faisant suivre leur cursus d'une agrégation de l'enseignement supérieur, soit en optant pour l'un des nouveaux masters "à finalité didactique». Ces deux voies proposent des contenus et une durée de formation strictement identiques, mais dans une temporalité différente. Actuellement, toutes les filières réservent peu de crédits à la formation générale et transversale, et les sciences humaines et sociales, comme outils d'analyse du système et des pratiques, y occupent une place réduite. Dans les débats en cours sur la réforme de la formation initiale des enseignants, qui vise à consolider la formation de tous les futurs enseignants en renforçant les collaborations entre les universités et les Hautes Écoles, les orientations qui se dessinent laissent peu de place aux enjeux de diversité. Toutefois, depuis 2001, le Conseil de la Communauté française

8. Il existe un référentiel des enseignants du secteur général des jeunes et un pour le secteur de la formation professionnelle et technique, qui se ressemblent, ainsi qu'un référentiel pour les directions scolaires. 
régissant la formation initiale des futurs enseignants prévoit treize compétences à développer, dont plusieurs rejoignent celles des référentiels français et québécois, en étant peu explicites sur la diversité ethnoculturelle, dont la $n^{\circ} 1$ (Mobiliser des connaissances en sciences humaines pour une juste interprétation des situations vécues en classe et autour de la classe et pour une meilleure adaptation aux publics scolaires), la no 7 (Développer les compétences relationnelles liées aux exigences de la profession), la no 8 (Mesurer les enjeux éthiques liés à sa pratique quotidienne), la no 11 (Entretenir un rapport critique et autonome avec le savoir scientifique passé et à venir) et la no 13 (Porter un regard réflexif sur sa pratique [...]).

En Suisse, tous les enseignants sont désormais formés dans les Hautes Écoles Pédagogiques (HEP) ${ }^{9}$, gérées par les cantons, dont les diplômes sont reconnus par la Conférence suisse des directeurs cantonaux de l'instruction publique pour l'ensemble du pays. S'il n'existe pas un curriculum «national» de formation des enseignants, la Conférence réunissant les responsables des HEP de Suisse romande et du Tessin a élaboré, en 2003, un "projet de référentiel pour la formation à l'enseignement» (Périsset Bagnoud, 2007), comportant onze compétences inspirées du référentiel québécois (Stierli et Tschopp, 2008). Il a été adapté dans les référentiels élaborés par les HEP cantonales. Une place plus importante qu'au Québec est donnée à la diversité ethnoculturelle et aux pratiques d'équité. Par exemple, dans le référentiel de la HEP du canton de Vaud (2004), on retrouve ces éléments dans au moins cinq des onze compétences. En plus de celles axées sur l'élève, telles que "prendre en compte la diversité culturelle et linguistique des élèves" (compétence 1.5) et "permettre à la classe d'exister comme un lieu ouvert à la pluralité des cultures" (compétence 1.6), le référentiel encourage les enseignants à la réflexivité critique, notamment sur leur propre enculturation ( "porter un regard critique sur ses propres origines et pratiques culturelles et sur son rôle social», compétence 1.3), mais aussi sur celle de l'école («établir des relations entre la culture prescrite dans le plan d'études et celle des élèves», compétence 1.4, et «discerner les valeurs en jeu dans ses interventions», compétence 3.1), ainsi que sur les pratiques de discrimination ("éviter toute forme de discrimination et de dévalorisation à l'égard des élèves, des parents et des collègues", compétence 3.6). Le référentiel rejoint celui des trois autres sociétés, mais demeure peu arrimé aux programmes de formation du personnel.

\section{Les contenus des programmes et des cours}

Plusieurs programmes de formation initiale des enseignants ont intégré des cours sur la diversité, mais la recherche montre une préparation insuffisante. En France, il y a peu d'enquêtes actualisées sur ce qui est effectivement enseigné dans les Écoles supérieures du professorat et de l'éducation en matière de «diversité». Globalement, les enseignants français s'estiment peu formés $(30 \%$ de moins que la moyenne de

9. Les Hautes Écoles Pédagogiques sont généralement non-universitaires, sauf dans certains cantons, notamment pour les enseignants du secondaire I et II. 
l'OCDE) sur les plans pédagogiques, des contenus d'enseignement et des pratiques de classe (Teaching and Learning International Survey, 2014). Les plus forts besoins exprimés concernent la prise en charge d'élèves à besoins spécifiques, les approches pédagogiques différenciées et l'enseignement en milieu multiculturel ou plurilingue. Un rapport public sur la formation continue pointe «l'urgence à renforcer [...] l'expertise pédagogique et éducative des personnels et des collectifs de travail» concernant «la prise en compte de leur hétérogénéité [des élèves], les principes d'une école inclusive» (Filâtre, 2016, p. 25). Si les textes ministériels exigent que «le professeur connai[sse] les éléments de sociologie et de psychologie lui permettant de tenir compte, dans le cadre de son enseignement, de la diversité des élèves et de leurs cultures" (Arrêté du 12 mai 2010 portant définition des compétences à acquérir par les professeurs, documentalistes et conseillers principaux d'éducation pour l'exercice de leur métier) et soit sensibilisé «à la lutte contre les discriminations [...] à la prévention et à la résolution non violente des conflits» (Article L721-2 du Code de l'éducation), la formation est éloignée de cet objectif et varie d'une École supérieure du professorat et de l'éducation à l'autre.

La formation initiale des enseignants privilégie les disciplines, au détriment des sciences sociales, de la pédagogie et de la réflexivité professionnelle. Sauf pour certains programmes ${ }^{10}$, le «tronc commun» est souvent le seul moment - très court, chargé, et tourné vers les savoirs fondamentaux - où les futurs professionnels découvrent les sciences sociales appliquées à l'éducation. Les éventuels enseignements transversaux relatifs à la diversité sont concentrés en première année, laquelle est consacrée au concours, ce qui accroît le traitement formel et utilitariste de ces questions par les étudiants, qui y voient une attente conformiste envers le discours de "neutralité», ou vont privilégier les contenus rentables pour l'épreuve (Paquay et al., 2012). En outre, le suivi des stages est très variable selon les Écoles supérieures du professorat et de l'éducation, et les pratiques afférentes à la diversité y trouvent rarement une place. Ces thématiques font peu partie des choix de mémoire des futurs personnels, et on relève des formes d'autocensure vis-à-vis des questions de racisme ou de discrimination, jugées institutionnellement trop sensibles (Dhume, El Massioui et Sotto, 2015). En fin de compte, ces questions ne sont guère des priorités et peuvent n'être pas du tout traitées dans les cours, sauf dans des cours optionnels ou chez quelques formateurs engagés (Meunier, 2013).

Au Québec, une cartographie de la formation initiale du personnel scolaire sur la diversité ethnoculturelle dans les universités montre un développement institutionnel disparate, mais une expansion significative de ce champ, liée aux nouveaux cours disciplinaires d'éthique et culture religieuse et du domaine de l'univers social (monde contemporain, histoire et éducation à la citoyenneté) (Larochelle-Audet et al, 2013). Par contre, les cours transversaux spécifiques sur la pluriethnicité (généralement de sociologie de l'éducation), mis en place au cours des années 1990, se sont maintenus

10. En Sciences économiques et sociales, ou dans la formation des Conseillers principaux d'éducation, qui exercent dans le second degré, en collège ou en lycée. 
et font partie du tronc commun de cours, souvent obligatoires dans la plupart des programmes de formation ${ }^{11}$. Plus de la moitié de ces cours sont entièrement consacrés à ces enjeux. Ils visent l'acquisition de savoirs essentiels communs (immigration, discriminations, rapports ethniques, balises politiques et juridiques), d'attitudes critiques (ex. : réflexivité, décentration) et de pratiques pertinentes (ex.: pour intervenir dans les situations de discrimination et d'accommodements raisonnables). L'analyse montre aussi des contenus adaptés aux réalités régionales (autochtones, réfugiés, etc.), des approches théoriques mixtes et des pédagogies variées.

Cependant, cette formation est non-systématique d'une région et d'un programme de formation à l'autre. La masse critique de professeurs permanents sur ces questions est concentrée à Montréal et certaines universités hors Montréal ${ }^{12}$ n'offrent aucun cours. De plus, certains programmes offrent un seul cours qui aborde tous les marqueurs de diversité sociale ${ }^{13}$, ce qui a pour effet de traiter superficiellement les réalités liées à l'immigration et aux rapports ethniques. La cartographie révèle ainsi un ancrage institutionnel ambigu de cet enseignement, qui tient de l'absence d'exigences et d'orientations ministérielles claires quant à sa légitimité et aux objectifs qu'il devrait viser. Cette ambiguïté se reflète dans l'arrimage inexistant entre formations fondamentale et pratique, et la faible coordination entre programmes et formateurs (plus «disciplinaires» ou "transversaux») au sein des universités. Les compétences visées étant peu relayées et évaluées dans les stages, ceci a des impacts pédagogiques sur certains étudiants, qui y consacrent moins de temps et d'intérêt (Potvin et Larochelle-Audet, 2016).

En Belgique francophone, l'application des ambitions ministérielles dans les programmes de formation initiale est très inégale d'une institution et d'un formateur à l'autre, et ne fait l'objet d'aucune évaluation systématique. Depuis 2001, le cursus de formation des futurs enseignants du primaire et du secondaire inférieur comporte 120 heures de «Connaissance socioculturelle générale», comprenant un cours de 30 heures intitulé Approche théorique et pratique de la diversité culturelle et de la dimension de genre, susceptible d'aborder les notions d'identité, de culture et d'immigration dans une optique de prévention des discriminations. Une étude exploratoire (Chasseur, 2016) révèle que ces cours donnent lieu à des traductions hétérogènes, et que très peu abordent toutes les dimensions d'une formation interculturelle et antidiscriminatoire complète.

11. Ils sont octroyés en $2^{\mathrm{e}}, 3^{\mathrm{e}}$ ou $4^{\mathrm{e}}$ année de formation initiale (Bachelor).

12. Et dans les régions qui ont fait l'objet de la Politique de régionalisation de l'immigration (1991), soit les universités Laval (Québec) et de Sherbrooke. Cette politique vise à encourager les nouveaux arrivants à s'établir à l'extérieur de la région métropolitaine de Montréal. Les réfugiés à titre humanitaire sont souvent installés à l'extérieur de la métropole.

13. Dans l'ensemble, les étudiants des programmes d'enseignement des langues secondes ont plus d'un cours obligatoire sur ces questions, ceux du primaire et du secondaire (toutes disciplines) ont un cours obligatoire de 45 heures, alors que la plupart de ceux en formation professionnelle et technique n'en recoivent aucun (Larochelle-Audet et al, 2013). 
Du côté de la formation des agrégés du secondaire supérieur, qui n’ont pas de cours équivalent, l'un des quatre axes de formation porte sur «l'appropriation des connaissances socioculturelles", avec 30 heures d'enseignement en sociologie générale de l'éducation, et une place réduite à la diversité ethnique. Par contre, il existe depuis 2004 une «formation à la neutralité» de vingt heures, qui vise à doter les futurs enseignants d'un cadre juridique général et à susciter une réflexion éthique et épistémologique sur les questions du vivre-ensemble. La nécessité de former les enseignants aux questions de citoyenneté, de vivre-ensemble et de prise en compte de la diversité (notamment "convictionnelle») semble devenue plus criante à la suite des attentats terroristes perpétrés par l'État Islamique en Europe depuis 2015. Mais, si cette urgence a débouché sur la création d'un cours obligatoire d'éducation philosophique et à la citoyenneté dans le secondaire ${ }^{14}$, les nouveaux besoins ainsi générés n'ont pas encore été répercutés dans les programmes de formation initiale des enseignants. Cette nouvelle obligation a plutôt ravivé les clivages philosophiques et les tensions entre réseaux d'enseignement (officiel et catholique) qui compliquent toute réforme sur ces questions.

En Suisse, les inventaires des programmes de formation sur la diversité culturelle des enseignants dans les différentes institutions de formation n'ont pas été mis à jour (Alleman-Ghionda, de Goumoëns et Perregaux 1999; Sieber et Bischoff, 2007). Ces enquêtes montraient que, si la formation interculturelle était partie intégrante de tous les cursus de formation des enseignants, son institutionnalisation et son contenu étaient très variables d'une institution et d'un canton à l'autre. Aujourd'hui, cette réalité semble perdurer, puisqu'aucun concept commun de formation n'a pu être élaboré (Ogay, 2018).

\section{Les effets de la formation sur la pratique}

Peu de recherches ont analysé jusqu'ici les impacts de cette formation sur la pratique enseignante dans ces quatre sociétés. Certaines enquêtes ayant évalué la préparation professionnelle des enseignants à agir en contexte de diversité dans différents pays ont mis en évidence des représentations souvent négatives de la diversité, et un sentiment fréquent de ne pas être assez formés pour aborder des questions jugées controversées (Teaching and Learning International Survey, 2014; Frankenberg et SiegelHawley, 2008; Hachfeld et al., 2011). Au Québec, le portrait de la formation a fait état des difficultés des formateurs universitaires à aborder, avec les futurs enseignants, certaines questions sensibles, qui leur paraissent litigieuses ou abstraites, comme les accommodements raisonnables, les droits et libertés, les enjeux identitaires et le racisme (Larochelle-Audet et al., 2013). Ces questions suscitent des résistances et se traduisent peu dans les pratiques en classe des enseignants du primaire et du secondaire, qui craignent de «déstabiliser le climat» (Potvin et McAndrew, 2010). Ces

14. Décret du 22 octobre 2015.

Volume XLVI: 2 - Automne 2018 
difficultés ont été soulevées même par les enseignants des disciplines concernées (ex. : Éthique et culture religieuse, Éducation à la citoyenneté), qui disent faire peu d'éducation au pluralisme et aux droits pour développer l'autonomie intellectuelle ou questionner les préjugés, les valeurs ou l'ordre politique (Courtine Sivane et Jutras, 2015; Demers et al., 2016; Falaize et Absalon, 2009; Jadot et al., 2017). L'enquête ICCS de 2009 (Schulz et al. 2010) sur les programmes d'éducation civique dans différents pays européens révèle que les enseignants développent surtout des connaissances théoriques et abstraites sur les droits (60\%), mais peu sur la participation des élèves dans l'école (19\%) ou sur la communauté (16\%), et encore moins des stratégies pour lutter contre le racisme et les discriminations (10\%) (Bozec, 2016). Les travaux québécois et belges, tout comme la consultation sur le programme d'Enseignement moral et civique en France (Ministère de l'Éducation Nationale, de l'Enseignement Supérieur et de la Recherche, 2015), montrent un décalage entre la formation reçue, les objectifs des programmes et les fortes attentes de formation.

\section{DISCUSSION CONCLUSIVE}

Cet article a mis au jour quelques différences et convergences sur la place de la diversité dans la formation enseignante de quatre sociétés de la francophonie. Leurs différents "statuts» de majorité (fragile dans le cas québécois) se reflètent dans leurs définitions respectives du "Nous» et dans leurs politiques de reconnaissance de la diversité. D'un côté, l'idéologie unitariste française et son occultation de ces questions, de l'autre, trois entités francophones insérées dans des fédérations polyethniques marquées respectivement par une histoire de rapports de pouvoirs entre groupes nationaux, qui ont des incidences sur la prise en compte des rapports ethniques en éducation.

Mais ces différences masquent des similarités, car partout on observe une tension entre tendances assimilationnistes - (ré)activée par les polarisations internationales et les enjeux identitaires/nationalistes propres à ces sociétés - et les politiques de reconnaissance. Sur certaines questions sensibles, comme le racisme et les discriminations, ramenées par l'actualité au cœur des enjeux du vivre-ensemble, on constate une frilosité à les nommer et à les introduire dans les politiques (Potvin, 2008; Dhume, 2010). Si la légitimité de ces problématiques et l'édiction d'une politique globale relative à la formation montre un écart à première vue maximal entre le Québec - qui dispose d'une politique assumée d'éducation interculturelle, avec une formation du personnel et un référentiel qui oriente les programmes - et les trois pays européens - qui possèdent des référentiels nommant explicitement les enjeux liés à la diversité - il existe un faible arrimage entre les ambitions décrétales et leur traduction effective dans la formation initiale des quatre sociétés. L'existence des cours varie d'un territoire, d'une filière, d'une institution ou d'un formateur à l'autre, tout comme la durée affectée à ces questions et la pertinence des contenus. Ces cours, bien qu'institutionnalisés, dépendent largement de l'implication individuelle 
des formateurs, qui font face à des résistances. Partout, l'approche disciplinaire domine la formation, au détriment des savoirs et méthodes des sciences humaines et sociales, pourtant déterminants dans la compréhension des problématiques de diversité, d'inégalités et de rapports entre minoritaires et majoritaires, et dans la réflexivité critique face à sa pratique. De plus, l'apport de connaissances prévaut sur les dimensions pratiques et questionnements éthiques. Dans les quatre contextes, les compétences à développer, leur reconnaissance et leur opérationnalisation en stage demeurent à clarifier et à actualiser.

Ce bref portrait comparatif montre que les dispositifs de formation doivent mieux arrimer les programmes, les cours et les référentiels de compétences sur les enjeux «interculturels», ainsi que la nécessité d'accentuer la place des sciences sociales dans la formation des acteurs scolaires sur ces questions. Enfin, la recherche comparative gagnerait à recueillir plus systématiquement dans ces quatre contextes des données autant auprès des formateurs d'enseignants (stratégies pédagogiques, contenus et compétences visées) qu'auprès des étudiants finissants (acquis et pertinence de la formation reçue), et de mesurer l'impact à long terme de ces enseignements sur les attitudes, les compétences professionnelles acquises, les pratiques effectives, ou les mécanismes de résistance des acteurs scolaires (Observatoire sur la formation à la diversité et l'Équité, recherche en cours).

\section{Références bibliographiques}

ADAM, I. (2011). Une gestion différenciée de la diversité. Les politiques d'intégration des personnes issues de l'immigration en Flandre, en Wallonie et à Bruxelles». Dans J. Ringelheim (dir.), La diversité culturelle en droit belge. Bruxelles, Belgique: Académia Bruylandt.

ALLEMAN-GHIONDA, C., de GOUMOËNS, C. et PERREGAUX, C. (1999). Formation des enseignants et pluralité linguistique et culturelle: entre ouvertures et résistances Rapport de valorisation. Programme national de recherche 33. Berne, Suisse: Direction du Programme national de recherche 33 (PNR 33) et Centre suisse de coordination pour la recherche en éducation (CSRE).

ARNESEN A.-L., BIRZEA C., DUMONT B. et al. (2009). Politiques et pratiques de l'enseignement de la diversité socioculturelle. Rapport d'enquête sur la formation initiale des enseignants à la diversité socioculturelle. Strasbourg, France: Éditions du Conseil de l'Europe. 
BOZEC, G. (2016). Éducation à la citoyenneté à l'école: politiques, pratiques scolaires et effets sur les élèves. Repéré à http://www.cnesco.fr/wp-content/uploads/2016/09/ Rapport_education_citoyennete.pdf

CHASSEUR, B. (2015). Gestion de la diversité et mise en place de l'intégration dans les écoles: cartographie et analyse de la formation des enseignants. Mémoire de master en Politiques économiques et sociales. Université catholique de Louvain.

Conférence intercantonale de l'instruction publique de la Suisse romande et du Tessin (2003). Déclaration relative aux finalités et objectifs de l'École publique. Repéré à http://www.ciip.ch/La-CIIP/Documents-officiels/Declarations-politiques

Conférence suisse des rectrices et recteurs des hautes écoles pédagogiques (2007). Recommandations relatives à la formation des enseignantes et enseignants aux approches interculturelles. Repéré à https://www.swissuniversities.ch/fileadmin/ swissuniversities/Dokumente/Kammern/Kammer_PH/Empf/071115_Empf_IKP_fr.pdf

COURTINE SINAVE, S. et JUTRAS, F. (2015). Éléments indicateurs de la laïcité dans le cadre de discussions d'enseignants sur l'éducation à la citoyenneté à l'école secondaire. McGill Journal of Education 50(1), 51-78.

DELORS, J. (1996). L'éducation: un trésor est caché dedans. Paris, France: Odile Jacob.

DEMERS, S., LEFRANÇOIS, D. et ÉTHIER, M.A. (2016) Les programmes québécois d'histoire et éducation à la citoyenneté en contexte de diversité. Dans M. Potvin, M.-P. Magnan et J. Larochelle-Audet (dir.), La diversité ethnoculturelle, religieuse et linguistique en éducation. Théorie et pratique (p. 110-127). Montréal, Québec: Fides.

DHUME, F. (2016). Comment l'antiracisme devint une «valeur de l'école». Diversité, $182,47-53$.

DHUME, F. (2012). La "diversité à l'école”: une occultation de l'ethnicisation et de la discrimination. Les Cahiers de Profession banlieue, 53-67.

DHUME, F. (2010). L'école face à la discrimination ethnoraciale: les logiques d'une inaction publique. Migrations Société, 131, 171-184.

DHUME, F. El MASSIOUI, N. et SOTTO, F. (2015). Former et enseigner sur la (non-) discrimination à l'école? Un enjeu politique incertain. Rapport pour le Défenseur des droits et l'Alliance pour la recherche sur les discriminations (ARDIS). Paris, France: ISCRA / Les Zégaux. 
DHUME, F, DUKIC, S., CHAUVEL, S. et PERROT, P. (2011). Orientation scolaire et discrimination. De l'(in)égalité de traitement selon «l'origine». Paris, France: La Documentation française.

FALAIZE, B. (2016). L'histoire à l'école élémentaire depuis 1945. Rennes, France: Presses universitaires de Rennes.

FALAIZE, B. et ABSALON, B. (2009). Enseigner l'histoire de l'immigration à l'école. Paris, France: Cité nationale de l'histoire de l'immigration et Institut national de recherche pédagogique.

FAURE, E. (1972). Apprendre à être. Repéré à http://unesdoc.unesco.org/images/0013/ 001329/132982f.pdf

FILÂTRE, D. (2016). Vers un nouveau modèle de formation tout au long de la vie. Repéré à http://www.ladocumentationfrancaise.fr/var/storage/rapports-publics/164000754. pdf

FRANKENBERG, E. et SIEGEL-HAWLEY, G. (2008). Are Teachers Prepared for Racially Changing Schools? Teachers Describe Their Preparation, Resources and Practices for Racially Diverse Schools. Los Angeles, CA: University of California.

HACHFELD, A. HAHN, A., SCHROEDER, S., ANDERS, Y., STANAT, P. et KUNTER, M. (2011). Assessing teachers' multicultural and egalitarian beliefs: The Teacher Cultural Beliefs Scale. Teaching and Teacher Education 27(6), 986-996.

Haute école pédagogique du canton de Vaud (2004). Formation des enseignantes et enseignants: Référentiel de compétences professionnelles. Repéré à https:// www.hepl.ch/files/live/sites/systemsite/files/interfilieres/referentiel-competences2015-hep-vaud.pdf

JADOT, E., VERHOEVEN, M. et DERBAIX B. (2017). Recherche-action portant sur l'Éducation à la citoyenneté démocratique et les droits humains dans l'enseignement en Fédération Wallonie-Bruxelles, Repéré à http://www.oejaj.cfwb. be/index.php?id $=18480$

KYMLICKA, W. (1998) Finding Our Way. Rethinking Ethnocultural Relations in Canada. Toronto, ON: Oxford University Press.

LABELLE, M. et SALÉE, D. (1999) La citoyenneté en question: l'État canadien face à l'immigration et à la diversité nationale et culturelle. Sociologie et sociétés, XXXI (2), 125-144. 
LANFRANCHI, A., PERREGAUX, C. \& THOMMEN, B. (2000). Pour une formation des enseignantes et enseignants aux approches interculturelles. Principaux domaines de formation - Propositions pour un curriculum de formation Indications bibliographiques. Berne: Conférence suisse des directeurs cantonaux de l'instruction publique (CDIP) (Rapport 60).

LANTHEAUME, F. (2011). La prise en compte de la diversité: émergence d'un nouveau cadre normatif ? Essai de généalogie et identification de quelques conséquences. Les dossiers des sciences de l'éducation. Repéré à http://journals. openedition.org/dse/1120.

LAROCHELLE-AUDET, J., BORRI-ANADON, C, Mc ANDREW, M. et POTVIN, M (2013) La formation initiale du personnel scolaire sur la diversité ethnoculturelle, religieuse et linguistique dans les universités québécoises: portrait quantitatif et qualitatif. Repéré à http://www.ceetum.umontreal.ca/documents/publications/ 2013/formation.pdf

LEVINE, M. V. (dir.). (1990). The Reconquest of Montreal: Language Policy and Social Change in a Bilingual City. Philadelphie, PA: Temple University Press.

LORCERIE, F. (1994). L'Islam dans les cours de «Langue et Culture d'origine»: le procès. Revue européenne des migrations internationales, 10(2), 5-43.

LORCERIE, F. (2012). France: Le rejet de l'interculturalisme. Studi Migrazione/ International Journal of Migration Studies, 186, 278-301.

MARTINIELLO M. et MANÇO A. (1993). Intercultural Education in French speaking Belgium: an overview of ideology, legislation and practice. European Journal of Intercultural studies, 4(2), 19-27.

Mc ANDREW, M. (2010). Les majorités fragiles et l'éducation: Belgique, Catalogne, Irlande du Nord, Québec. Montréal, Québec: Presses de l’Université de Montréal.

Mc ROBERTS, K. (1999). Un pays à refaire. L'échec des politiques constitutionnelles canadiennes. Montréal, Québec: Boréal.

MEUNIER, O. (2013). Un rapport difficile à la diversité dans l'école de la République. Approche sociohistorique du modèle français. Revue internationale d'éducation de Sèvres, 63, 89-98.

MOREL, S. (2002). École, territoire et identités. Les politiques publiques françaises à l'épreuve de l'ethnicité. Paris, France: L'Harmattan.

Ministère de l'Éducation du Québec (1985). Rapport du comité sur l'école québécoise et les communautés culturelles. Québec, Québec: Gouvernement du Québec. 
Ministère de l'Éducation du Québec (1998). Une école d'avenir: Politique d'intégration scolaire et d'éducation interculturelle. Québec, Québec: Gouvernement du Québec.

Ministère de l'Éducation du Québec (2001). La formation à l'enseignement, les orientations et les compétences professionnelles. Québec, Québec: Gouvernement du Québec.

Ministère de l'Éducation et de l'Enseignement Supérieur (2017). Politique de la réussite éducation. Le plaisir d'apprendre, la chance de réussir. Québec: Gouvernement du Québec.

Ministère de l'Education Nationale, de l'Enseignement Supérieur et de la Recherche (2015). Synthèse de la consultation nationale sur les projets de programmes d'enseignement moral et civique. Repéré à http://cache.media.eduscol.education. fr/file/Actu_2015/74/8/Rapport-de-synthese-de-la-consultation-nationale-sur-lesprojets-de-programmes-denseignement-moral-et-civique_476748.pdf

Ministère de l'Education nationale (2013). Référentiel des compétences professionnelles des métiers du professorat et de l'éducation. Repéré à http://www.education.gouv. fr/cid73215/le-referentiel-de-competences-des-enseignants-au-bo-du-25-juillet-2013. html

Ministère des Communautés culturelles et de l'Immigration (1990). L'Énoncé de politique en matière d'immigration et d'intégration des immigrants. Montréal, Québec: Gouvernement du Québec.

Observatoire sur la formation à la diversité et l'Équité (en cours). Enquête sur l'acquisition des compétences interculturelles et inclusives du personnel scolaire au Québec.

OGAY, T. (2012). Pluralité des langues et des cultures: quatre expériences institutionnelles, une même quête d'équilibre dans la tension entre égalité et diversité. Alterstice, 2(1), 9-16.

OGAY, T. (2018). La reconnaissance de la diversité ethnoculturelle dans la formation des enseignants en Suisse romande: la diversité comme objet et contrainte. Dans F. Lorcerie (dir.), Éducation et diversité. Les fondamentaux de l'action. Paris, France: Presses universitaires de Rennes. Manuscrit soumis pour publication.

OUELLET, F. (1991). L'éducation interculturelle. Essai sur le contenu de la formation des maîtres. Paris, France: L'Harmattan.

PAQUAY, L., ALTET M., CHARLIER E. et PERRENOUD P. (2012), Former des enseignants professionnels. Quelles stratégies? Quelles compétences? Bruxelles, Belgique: De Boeck. 
PÉRISSET BAGNOUD, D. (2007). Les référentiels de compétences, profils attendus de la professionnalité enseignante. Acteurs et points de vue en Suisse romande. In L. Talbot et M. Bru (dir.), Des compétences pour enseigner: Entre objets sociaux et objets de recherche (p. 87-104). Rennes, France: Presses de l’Université de Rennes.

POTVIN, M. (2018). De l'interculturel à l'inclusion au Québec: des changements de paradigmes? Dans C. Borri-Anadon, G. Gonçalves, S. Hirsch et J. Queiroz Odinino (dir.), La formation des éducateurs en contexte de diversité: une perspective comparative Québec-Brésil. Blue Mounds, WI: Deep University Press.

POTVIN, M. (2016). L'éducation au pluralisme: la centralité des sciences sociales. Philosophiques, 43(2), 481-488.

POTVIN, M. ([2010]2016, $2^{\mathrm{e}}$ ed.). Interethnic Relations and Racism in Quebec. Dans C. Kirkey, R. Jarrett et S. Gervais (dir), Quebec Questions. Québec Studies for the $21^{\text {st }}$ Century (p. 271-296). London: Oxford University Press.

POTVIN, M. et LAROCHELLE-AUDET, J. (2016). Les approches théoriques sur la diversité ethnoculturelle en éducation et les compétences essentielles du personnel scolaire. Dans M. Potvin, M.-O. Magnan et J. Larochelle-Audet. (dir.), La diversité ethnoculturelle, religieuse et linguistique en éducation (p. 110-127). Théorie et pratique. Montréal, Québec: Fides.

POTVIN, M., BORRI-ANADON, C., LAROCHELLE-AUDET, J. ARMAND, F., BECK, I.-A. Cividini, M., ... Steinbach, M. (2015). Rapport sur la prise en compte de la diversité ethnoculturelle, religieuse et linguistique dans les orientations et compétences professionnelles en formation à l'enseignement. Repéré à http://collections.banq. qc.ca/ark:/52327/bs2482627

POTVIN, M. et Mc ANDREW, M. (2010). Racisme et antiracisme en milieu scolaire francophone à Montréal: politiques, pratiques et perceptions d'intervenants. Dans G. Thésée, N. Carignan et P. Carr (dir.), Les faces cachées de la recherche interculturelle (p. 163-183). Paris, France: L'Harmattan.

POTVIN, M. (2008). Racisme et discours public commun au Québec. Dans Stephan Gervais, Dimitrios Karmis et Diane Lamoureux (dir.), De tricoté serré à métissé serré ? La culture publique commune en débats (p. 227-248). Québec, Québec: Les Presses de l'Université Laval. https://www.researchgate.net/ publication/314207102_Chapitre_10_Racisme_et_discours_public_commun_ au_Quebec.

REY-VON ALLMEN, M. (2011). The intercultural perspective and its development through cooperation with the Council of Europe. Dans C. A. Grant et A. Portera (dir.), Multicultural Education. Enhancing global interconnectedness (p. 33-48). New York, NY: Routledge. 
SAYAD, A. (2014). L'école et les enfants de l'immigration. Paris, France: Seuil.

SCHULZ, W. et al. 2010. ICCS 2009. International report: civic knowledge, attitudes and engagement among lower secondary school students in 38 countries. Amsterdam: International Association for the Evaluation of Educational Achievement (IEA).

SIEBER, P. et BISCHOFF, S. (2007). Examen de la situation actuelle de la pédagogie interculturelle au sein des hautes écoles pédagogiques et des établissements de formation des enseignants de Suisse. Repéré à https://www.swissuniversities. ch/fileadmin/swissuniversities/Dokumente/Kammern/Kammer_PH/ Dok/2007_Untersuchungsbericht_IKP_fr.pdf

STIERLI, E. et TSCHOPP, G. (2008). Quel référentiel de compétences professionnelles pour les enseignants? Dans J. Weiss (dir.), Quelle évaluation des enseignants au service de l'école? (pp. 45-51). Neuchâtel, Suisse: Institut de recherche en documentation pédagogique.

Teaching and Learning International Survey (2014). Une perspective internationale sur l'enseignement et l'apprentissage. Repéré à https://www.oecd-ilibrary.org/ education/resultats-de-talis-2013_9789264214293-fr

TAYLOR, C. (1992). Multiculturalism: Examining the Politics of Recognition. Princeton, $\mathrm{NJ}$ : Princeton University Press.

TREMBLAY, S. (2016). Les écoles religieuses et ethno spécifiques: histoire, enjeux et réalités. Dans M. Potvin, M.-O., Magnan et J. Larochelle-Audet. (dir.), La diversité ethnoculturelle, religieuse et linguistique en éducation. Théorie et pratique ( $\mathrm{p}$. 60-67). Montréal, Québec: Fides.

UNESCO (Organisation des Nations Unies pour l'Éducation, la Science et la Culture) (2009). Principes directeurs pour l'inclusion dans l'éducation. Paris: UNESCO.

UNESCO (2015) Éducation à la citoyenneté mondiale. Préparer les apprenants aux défis du XXIe siècle. Paris: UNESCO.

VERHOEVEN, M. (2003). Modèles d'intégration nationaux, dynamiques d'établissements et processus identitaires en contextes multiculturels: regards croisés Angleterre - Communauté française de Belgique. Revue Française de Pédagogie, 144, 9-17.

ZOÏA, G. (2015). Former les enseignants à la diversité culturelle. Diversité, 177, 137-142. 